\title{
Compléments à l'étude des rongeurs (Mammalia, Rodentia, Cricetidae, Eomyidae, Sciuridae) du gisement karstique de Blanquatère 1 (Miocène moyen, sud de la France)
}

\author{
Jean-Pierre AGUILAR \\ Jacques MICHAUX \\ Université Montpellier 2, UMR-CNRS 5554, \\ Institut des Sciences de l'Évolution, place E. Bataillon, cc 064, \\ F-34095 Montpellier cedex 05 (France) \\ jean-pierre.aguilar@univ-montp2.fr \\ jacques-jules.michaux@univ-montp2.fr \\ Bertrand AUNAY \\ BRGM Réunion, \\ 5 rue Sainte-Anne, BP 906, 97478 Saint Denis cedex (La Réunion) \\ b.aunay@brgm.fr \\ Marc CALVET \\ Université de Perpignan, Faculté des Lettres et Sciences humaines, \\ Médi-Terra, 52 avenue Paul Alduy, F-66025 Perpignan cedex (France) \\ calvet@univ-perp.fr \\ Vincent LAZZARI \\ Université de Poitiers, UMR-CNRS 6046, IPHEP, \\ 40 avenue du Recteur Pineau, F-86022 Poitiers (France) \\ vincent.lazzari@univ-poitiers.fr
}

MOTS CLÉS

Mammalia,

Rodentia,

Cricetidae,

Eomyidae,

Sciuridae,

karst,

rongeurs,

Miocène moyen, sud de la France.
Aguilar J.-P., Michaux J., Aunay B., Calvet M. \& Lazzari V. 2010. - Compléments à l'étude des rongeurs (Mammalia, Rodentia, Cricetidae, Eomyidae, Sciuridae) du gisement karstique de Blanquatère 1 (Miocène moyen, sud de la France). Geodiversitas 32 (3): 515-533.

\section{RÉSUMÉ}

Le gisement karstique de Blanquatère 1 a livré une microfaune d'âge Miocène moyen, abondante (plus de 6300 dents) et très diversifiée en rongeurs avec 33 espèces reconnues, parmi lesquelles plusieurs espèces nouvelles de Cricetidae et de Gliridae. Le présent travail complète l'étude initiale par la description de Cricetidae non décrits jusqu'ici, Eumyarion, Democricetodon, Pseudofahlbuschia, des Eomyidae Ligerimys et Keramidomys et des Sciuridae Spermophilinus et Heteroxerus. Cette faune, très riche, fait de cette localité une référence pour le début du Miocène moyen (MN 4/MN 5) en Europe sud-occidentale. La faune inclut des taxons nouveaux et allochtones avec, en particulier, la présence d'un représentant des Myocricetodontinae (Dakkamys sp.), qui résulte d'une probable 


\section{KEY WORDS \\ Mammalia, \\ Rodentia, \\ Cricetidae, \\ Eomyidae, \\ Sciuridae, \\ karstic filling, rodents, \\ Middle Miocene, southern France.}

immigration suite à la fermeture orientale de la Téthys au cours du Langhien. C'est aussi actuellement le site le plus ancien dans lequel cohabitent Ligerimys et Keramidomys. Enfin, cette localité souligne encore une fois l'intérêt des remplissages karstiques, susceptibles d'enregistrer des occurrences de courte durée de taxons consécutives à des événements géologiques ou climatiques.

\begin{abstract}
Addition to the study of rodents (Mammalia, Rodentia, Cricetids, Eomyids, Sciurids) from the karstic locality of Blanquatère 1 (Middle Miocene, southern France).

Blanquatere 1 is the filling of a fissure opened in a limestone that had already provided a rich fauna (more than 6300 isolated teeth, 33 species) of small mammals including mainly rodents among which several new species of Cricetidae and Gliridae, referred to the Middle Miocene. The present paper brings an addition to this fauna with the description of the Cricetidae Eumyarion, Democricetodon, and Pseudofahlbuschia, of the Eomyidae Ligerimys et Keramidomys, and of the Sciuridae Spermophilinus and Heteroxerus. This highly diverse fauna must be definitely considered as a reference for the first part of the Middle Miocene (MN 4/5) in South Western Europe. It testifies of the occurrence of both new taxa and allochtonous ones, as the gerbillid Dakkamys sp. the occurrence of which being likely related to the closure of the Tethys in the Middle East during the Langhian. The co-occurrence of Ligerimys and Keramidomys is also documented as the oldest one. This fauna also illustrates the interest of karstic fissure fillings when they are exceptionally rich: they can deliver remains of species either very rare or of short duration.
\end{abstract}

\section{INTRODUCTION}

Le gisement karstique de Blanquatère 1 (Fig. 1) a livré plus de 6300 dents (premières et secondes molaires inférieures et supérieures) de rongeurs. La faune, datée du début du Miocène moyen (limite MN 4/ MN 5) avec un âge estimé vers $16 \mathrm{Ma}$ (Lazzari \& Aguilar 2007), est remarquable par sa diversité en rongeurs (33 espèces) dont plusieurs travaux ont rendu compte: ils se rapportent à la sous-famille des Cricetodontinae Stehlin \& Schaub, 1951, avec le genre Megacricetodon Fahlbusch, 1964 (Lazzari \& Aguilar 2007), à la sous-famille des Myocricetodontinae Lavocat, 1961 avec le genre Dakkamys Jaeger, 1977 (Lazzari et al. 2007) ainsi qu’à la famille des Gliridae Thomas, 1897 (Aguilar \& Lazzari 2006). Le présent travail apporte la description des autres rongeurs reconnus dans la faune.

Les mensurations des dents ont été effectuées sous une loupe binoculaire Wild, à l'aide d'une platine de mesures Leica et sont exprimées en millimètres. Le matériel est déposé dans les collections de l'Université Montpellier 2. Le sigle utilisé est BLQ 1.

Les terminologies utilisées pour la description des dents de Cricetidae Rochebrune, 1883, de Gliridae et de Sciuridae Gray, 1821 sont respectivement celles proposées par Mein \& Freudenthal (1971), Daams (1999) et Cuenca Bescós (1988).

\section{SYSTÉMATIQUE}

\author{
Ordre RODENTIA Bowdich, 1821 \\ Famille CRICETIDAE Rochebrune, 1883 \\ Sous-famille CRICETODONTINAE \\ Stehlin \& Schaub, 1951
}

Genre Megacricetodon Fahlbusch, 1964

Nous rappellerons ici l'existence de trois espèces du genre Megacricetodon: $M$. «collongensis-gersii» (voir 


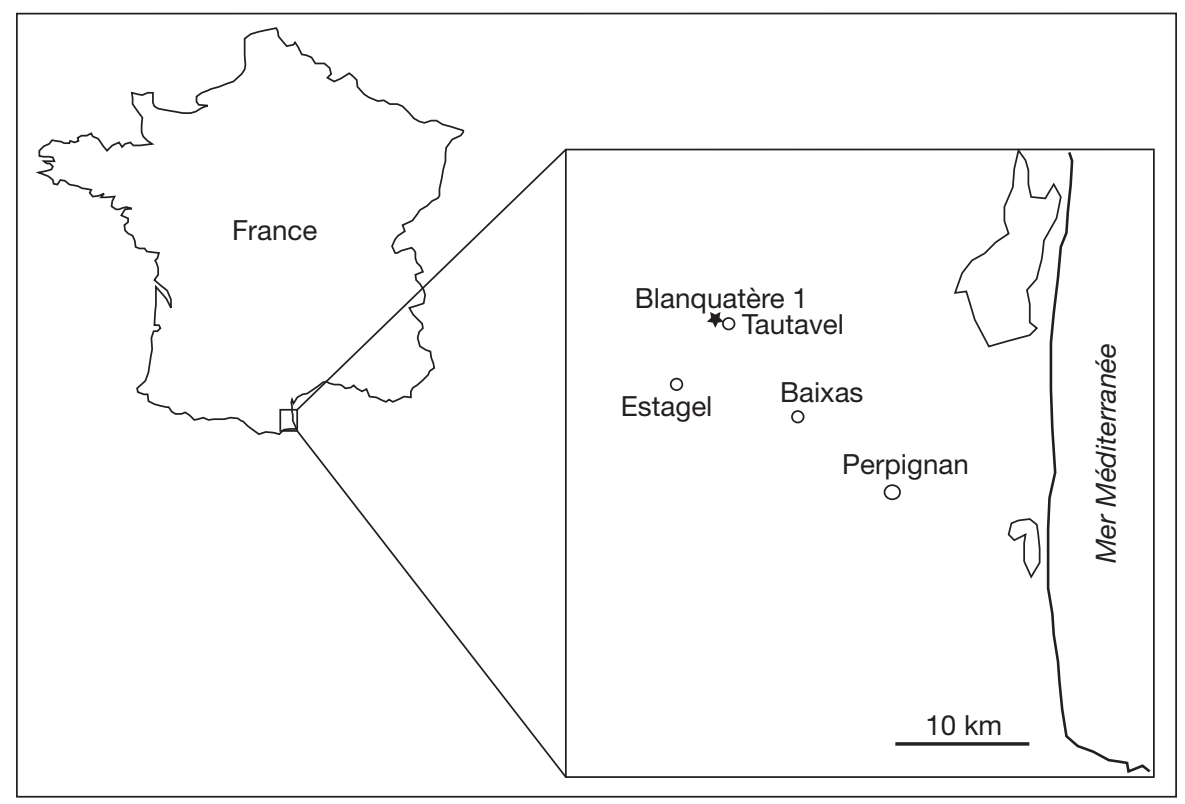

FIG. 1. - Localisation du gisement de Blanquatère 1 (Pyrénées-Orientales, France).

Aguilar 1995), M. tautavelensis Lazzari \& Aguilar, 2007 et M. aunayi Lazzari \& Aguilar, 2007.

\section{Genre Pseudofahlbuschia}

Freudenthal \& Daams, 1988

Pseudofablbuschia aff. jordensis

Freudenthal \& Daams, 1988

(Fig. 2; Tableau 1)

Matériel et dimensions. — Dents isolées (BLQ 1 $n^{\circ} 1359$ à 1432). Dimensions: voir Tableau 1.

\section{DESCRIPTION}

\section{Molaires inférieures}

Les molaires inférieures montrent une morphologie proche de celle de P. catalaunica Aguilar, 1999 des gisements de Baixas 200 et 201 (Aguilar 1999) mais quelques différences apparaissent toutefois: le rapport $\mathrm{L} / \mathrm{l}$ des $\mathrm{m} 1$ est de 1,50 alors que chez P. catalaunica, il est de 1,65 et il n'y a pas d'éperon labial sur l'antérolophulide. Sur $3 \mathrm{~m} 1$, le mésolophide est plus développé.
TABleau 1. - Mensurations (en mm) des molaires de Pseudofahlbuschia aff. jordensis Freudenthal \& Daams, 1988 du gisement de Blanquatère 1.

\begin{tabular}{lrrrrrrrr}
\hline & & \multicolumn{3}{c}{ Longueur } & & \multicolumn{3}{c}{ Largeur } \\
\cline { 3 - 5 } \cline { 7 - 9 } & $\mathbf{N}$ & min & moy & max & & min & moy & max \\
\hline m1 & 14 & 1,63 & 1,81 & 2,01 & & 1,05 & 1,21 & 1,33 \\
m2 & 12 & 1,45 & 1,59 & 1,72 & & 1,29 & 1,35 & 1,44 \\
m3 & 2 & 1,35 & & 1,39 & & 1,06 & & 1,15 \\
M1 & 18 & 1,97 & 2,09 & 2,35 & & 1,27 & 1,38 & 1,51 \\
M2 & 26 & 1,36 & 1,50 & 1,64 & & 1,23 & 1,35 & 1,55 \\
M3 & 2 & 1,12 & & 1,15 & & 1,09 & & 1,14 \\
\hline
\end{tabular}

\section{Molaires supérieures}

Aux molaires supérieures, l'éperon labial de l'antérolophule n'est bien développé que sur une dent et le mésolophe est de longueur moyenne sur deux M1, alors qu'il est pratiquement absent dans la population type. Le postérosinus est toujours très réduit et n'est absent que sur 1 seule dent non usée. Sur M2, le métalophule est postérieur (12 dents) ou absent (1), sur les autres il est transverse à proverse. M1 et M2 n'ont pas de double protolophule. Cinq M1 et six M2 montrent un éperon postérieur du paracône plus ou moins développé. 
A

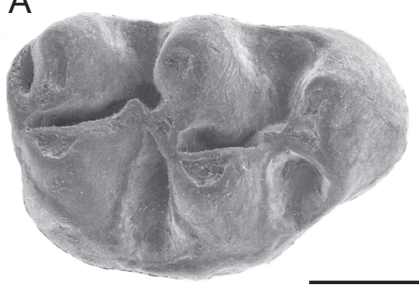

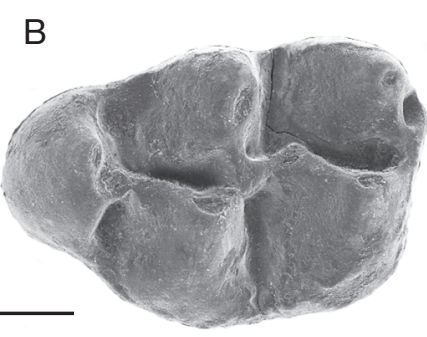

C

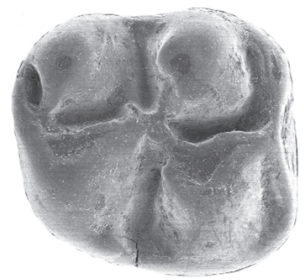

D

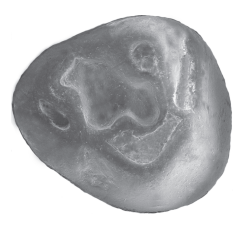

$\mathrm{E}$
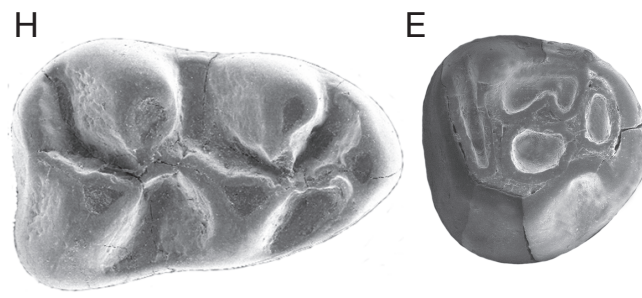

FIG. 2. - Molaires de Pseudofahlbuschia aff. jordensis Freudenthal \& Daams, 1988 de Blanquatère 1 (Pyrénées-Orientales, France) (mensurations en mm): A, M1 dex., 2,06 × 1,37 (BLQ $1 \mathrm{n}^{\circ}$ 1392); B, M1 sen., 2,17 × 1,42 (BLQ $1 \mathrm{n}^{\circ}$ 1387); C, M2 dex., 1,46 × 1,35 (BLQ $1 n^{\circ}$ 1405); D, M3 dex., 1,12 × 1,09 (BLQ $1 n^{\circ}$ 1431); E, M3 sen., 1,15 × 1,14 (BLQ $\left.1 \mathrm{n}^{\circ} 1432\right)$; F, m2 dex., 1,62 × 1,44 (BLQ 1 $\mathrm{n}^{\circ}$ 1374); G, m1 sen., 2,00 × 1,33 (BLQ 1 n 1359); H, m1 dex., 2,01 × 1,26 (BLQ $1 \mathrm{n}^{\circ}$ 1360). Échelle: $1 \mathrm{~mm}$.

Deux M3 ont été attribuées à cette espèce car elles n'ont pas d'antérolophe lingual, ce qui les différencie nettement des M3 de Democricetodon Fahlbusch, 1964. Il n'est pas impossible que certaines M3 du genre Democricetodon appartiennent au genre Pseudofahlbuschia car les M3 figurées par Freudenthal \& Daams (1988) ne montrent pas de différences particulières.

\section{DisCUSSION}

Cette population de Pseudofablbuschia présente des caractères qui la différencient à la fois de $P$. catalaunica des gisements de Baixas 200 et 201 (Aguilar 1999) et de $P$. jordensis du gisement ibérique de Villafeliche 4A (Freudenthal \& Daams 1988). Les caractères morphologiques les plus significatifs sont: le métalophule des M2 qui, dans près de la moitié des cas, est postérieur alors que dans les deux autres populations il est majoritairement transverse, et l'absence d'éperon sur l'antérolophulide des $\mathrm{m} 1$. Le rapport $\mathrm{L} / \mathrm{l}$ des $\mathrm{m} 1$ $(1,50)$ est plus proche de celui observé chez P. jordensis $(1,58)$ que de celui observé chez $P$. catalaunica $(1,65)$, il en est de même pour les dimensions des molaires qui sont voisines de celles de $P$. jordensis et inférieures à celles de $P$. catalaunica (Fig. 3).
Les $\mathrm{m} 1$ montrent également une morphologie proche de celle de D. affinis (Schaub, 1925) de La Grive $M$, qui présente un antéroconide souvent bien distinct de la première paire de tubercules contrairement aux autres populations de Democricetodon recueillies en Languedoc-Roussillon mais pour qui le rapport $\mathrm{L} / \mathrm{l}$ est de 1,45 . Toutefois, les molaires supérieures sont nettement différentes, par l'absence de double protolophule sur M1-2, la présence d'un éperon postérieur du paracône, des tubercules plus massifs et plus hauts, et des dimensions moyennes légèrement supérieures.

Van der Meulen et al. (2004) mettent en synonymie les genres Fablbuschia Mein \& Freudenthal, 1971, Pseudofahlbuschia Freudenthal \& Daams, 1988 et Renzimys Lacomba, 1983 avec le genre Democricetodon, synonymie contestée par Freudenthal (2006). La synonymie entre Democricetodon et Fablbuschia avait déjà été proposée par Aguilar (1981) et nous ne pouvons que souscrire à la proposition de Van der Meulen et al. (2004), en revanche, les genres Renzimys et Pseudofahlbuschia présentent quelques caractères morphologiques dentaires qui permettent de les différencier du genre Democricetodon. Ces deux genres ont la particularité d'avoir une $\mathrm{m} 1$ qui présente 

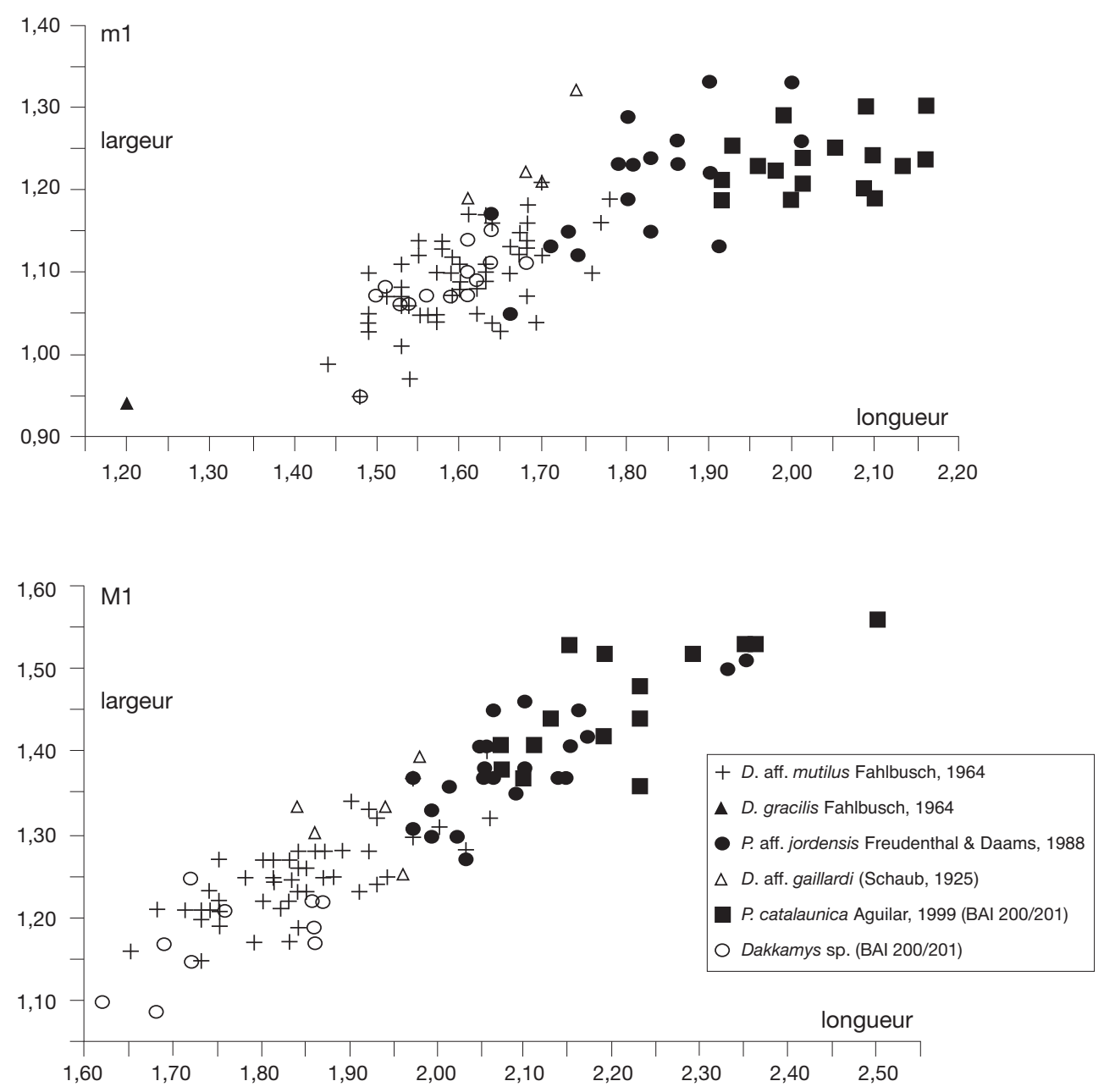

FIG. 3. - Diagrammes bivariés de répartition longueur/largeur des mesures de la première molaire inférieure (m1) et supérieure (M1) des Democricetodon Fahlbusch, 1964 et du Pseudofahlbuschia Freudenthal \& Daams, 1988 de Blanquatère 1 (Pyrénées-Orientales, France). Comparaison avec les populations de Baixas (BAl) 200 et 201.

une morphologie proche de celle du genre Megacricetodon et non de celle du genre Democricetodon. Le prélobe est bien différencié avec un antérocônide bien distinct de la première paire de tubercules et plus particulièrement du métaconide; le rapport L/l des espèces Renzimys lacombai Freudenthal \& Daams, 1988 et $P$. catalannica est supérieur à celui généralement observé chez la plupart des espèces du genre Democricetodon; le rapport observé chez l'espèce type $P$. jordensis et chez $P$. aff. jordensis de Blanquatère 1 est toutefois comparable à celui des espèces du genre Democricetodon. Aux molaires supérieures, nous noterons, du moins pour les deux populations du sud de la France, l'absence constante de double protolophule, la présence éventuelle d'un éperon labial au paracône chez $P$. aff. jordensis ainsi que la structure plus massive des tubercules.

Aguilar (1999) pensait qu'il pouvait exister un diachronisme dans l'apparition du genre Pseudofablbuschia, en France et en Péninsule ibérique, au cours du Miocène moyen, mais la découverte de $P$. aff. jordensis dans le gisement de Blanquatère 1, 
TABleau 2. - Mensurations (en $\mathrm{mm}$ ) des molaires de Democricetodon gracilis Fahlbusch, 1964 du gisement de Blanquatère 1 (Pyrénées-Orientales, France).

\begin{tabular}{|c|c|c|c|c|c|c|c|}
\hline & \multirow[b]{2}{*}{$\mathbf{N}$} & \multicolumn{3}{|c|}{ Longueur } & \multicolumn{3}{|c|}{ Largeur } \\
\hline & & $\min$ & moy & $\max$ & $\min$ & moy & $\max$ \\
\hline $\mathrm{m} 1$ & 1 & & 1,20 & & & 0,94 & \\
\hline $\mathrm{m} 2$ & 15 & 1,00 & 1,09 & 1,20 & 0,80 & 0,89 & 0,99 \\
\hline $\mathrm{M} 2$ & 5 & 1,12 & 1,20 & 1,25 & 0,99 & 1,02 & 1,04 \\
\hline
\end{tabular}

TABleau 3. - Mensurations (en mm) des molaires de Democricetodon aff. mutilus Fahlbusch, 1964 du gisement de Blanquatère 1 (Pyrénées-Orientales, France). N, nombre de dents dont la longueur/largeur est mesurable.

\begin{tabular}{lcccccccc}
\hline & & \multicolumn{3}{c}{ Longueur } & & \multicolumn{3}{c}{ Largeur } \\
\cline { 3 - 5 } \cline { 7 - 8 } & $\mathbf{N}$ & min & moy & max & & min & moy & max \\
\hline m1 & $56 / 55$ & 1,44 & 1,60 & 1,78 & & 0,95 & 1,09 & 1,19 \\
m2 & 54 & 1,18 & 1,39 & 1,51 & & 1,05 & 1,19 & 1,31 \\
m3 & 47 & 1,16 & 1,29 & 1,43 & & 0,99 & 1,07 & 1,21 \\
M1 & $49 / 50$ & 1,65 & 1,84 & 2,08 & & 1,15 & 1,25 & 1,40 \\
M2 & 57 & 1,24 & 1,41 & 1,55 & & 1,16 & 1,25 & 1,35 \\
M3 & 44 & 0,91 & 1,05 & 1,15 & & 0,98 & 1,08 & 1,19 \\
\hline
\end{tabular}

dont l'âge est plus ancien que ceux de Baixas 200 et 201, infirme cette hypothèse. Comme nous le verrons plus loin, la composition faunique du gisement de Blanquatère 1 permet de mettre en corrélation ce site avec les sites ibériques de Villafeliche 4A, Valdemoros 3D, attribués aux zones D0 à D2 (Van der Meulen \& Daams 1992) et dans lesquels le genre Pseudofahlbuschia est signalé. En conséquence, la présence de ce genre paraît sensiblement synchrone dans les deux régions.

Genre Democricetodon Fahlbusch, 1964

Democricetodon gracilis Fahlbusch, 1964 (Fig. 4A-C; Tableau 2)

Matériel et Dimensions. - Dents isolées (BLQ 1 $n^{\circ} 2122$ à 2142). Dimensions: voir Tableau 2.

\section{DESCRIPTION}

\section{Molaires inférieures}

L'unique $\mathrm{m} 1$, de très petite taille (Fig. 3), montre un petit antéroconide dont la partie linguale rejoint le métaconide et un court mésolophide. Sur les m2, le mésolophide est de longueur variable, absent ou à peine marqué (7) court (5), ou moyennement long (3).

\section{Molaires supérieures}

Elles ne sont représentées que par des M2 qui montrent un double protolophule et un métalophule proverse. Le mésolophe est plus développé que sur les molaires inférieures, il est toujours long et atteint le bord de la couronne. Le sinus est bordé par une crête cingulaire plus ou moins forte qui se développe à partir du flanc de l'hypocône.

\section{DisCUSSION}

La présence, au sein du matériel attribué au genre Democricetodon, de quelques molaires singulières par leur petite taille (Figs 3; 5) présentant une morphologie et des dimensions comparables à celles de $D$. gracilis des gisements de Langenmoosen et de Sandelzhausen (Falbusch 1964; Moser et al. 2009), témoigne de l'existence de cette espèce au sein du gisement. Les molaires de Blanquatère 1 ont toutefois des dimensions inférieures à celles de la population de D. gracilis de Vieux-Collonges (Maridet 2002). Nous noterons que cette espèce est assez rare dans ces deux gisements où le genre Democricetodon est pourtant abondamment représenté.

\section{Democricetodon aff. mutilus Fahlbusch, 1964}

(Fig. 4D-O; Tableau 3)

Matériel et Dimensions. - Près de 1500 dents isolées. Dimensions: seul un certain nombre de molaires ont été mesurées, voir Tableau 3.

\section{DESCRIPTION}

\section{Molaires inférieures}

$\mathrm{m} 1$. L'antéroconide est presque toujours bien distinct de la première paire de tubercules mais sur quelques dents, le métaconide est proche de ce dernier. Le développement du mésolophide est variable, il est généralement court, mais il peut atteindre une longueur moyenne, ou être pratiquement absent, rarement long. Le sinuside, dirigé vers l'avant est souvent bordé par une crête cingulaire plus ou moins importante. 

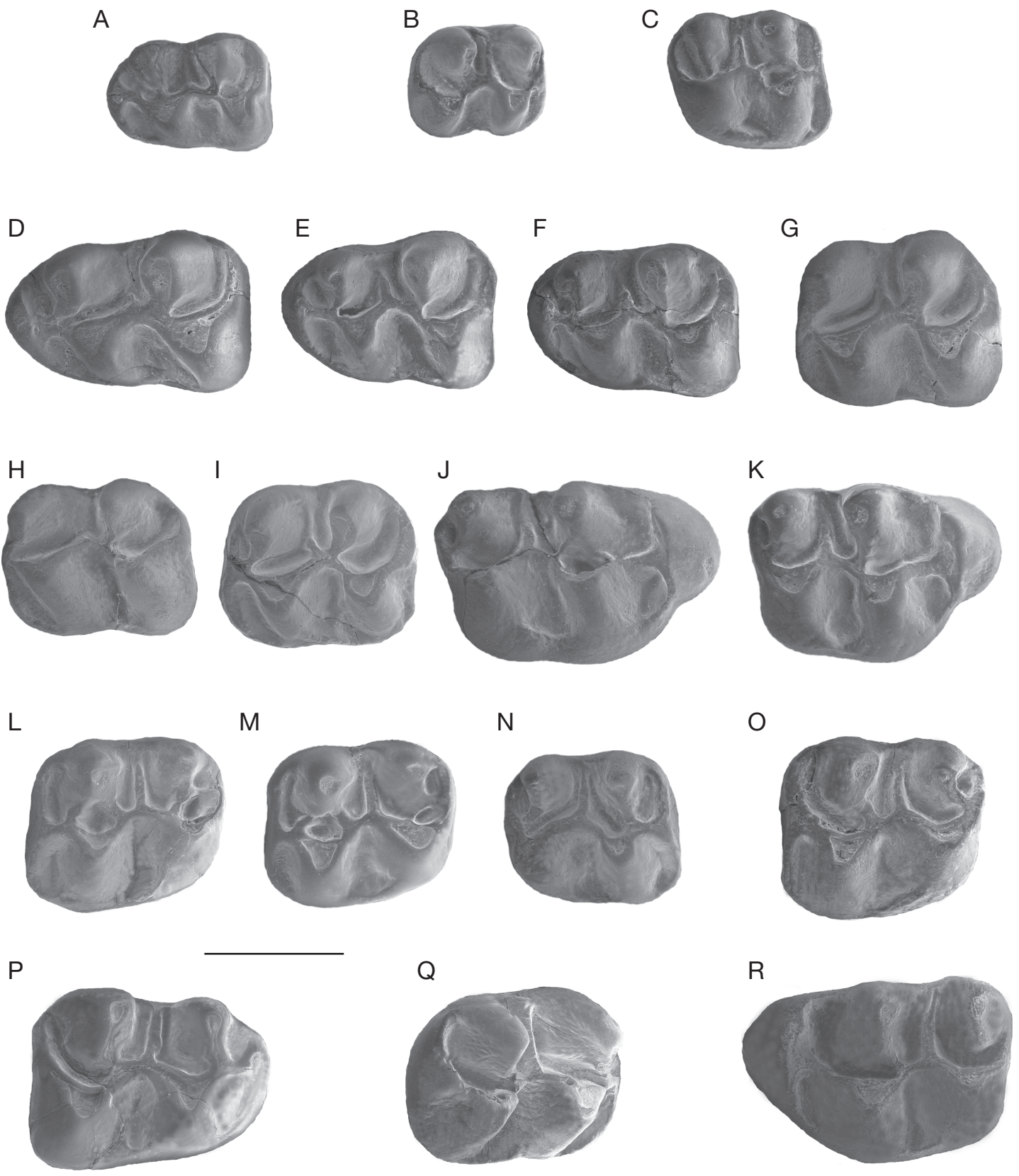

FIG. 4. - Molaires de Blanquatère 1 (Pyrénées-Orientales, France) (mensurations en mm): A-C, D. gracilis Fahlbusch, 1964; D-O, Democricetodon aff. mutilus Fahlbusch, 1964; P-R, D. aff. gaillardi (Schaub, 1925); A, m1 sen., 1,20 $\times 0,94$ (BLQ $1 \mathrm{n}^{\circ} 2122$ ); B, $\mathrm{m} 2$ dex., 1,00 × 0,84 (BLQ $\left.1 \mathrm{n}^{\circ} 2123\right) ; \mathbf{C}, \mathrm{M} 2$ dex., 1,19 × 0,99 (BLQ $\left.1 \mathrm{n}^{\circ} 2138\right) ; \mathbf{D}$, m1 sen., 1,77 × 1,16 (BLQ $\left.1 \mathrm{n}^{\circ} 2160\right) ; \mathbf{E}, \mathrm{m} 1$ sen., 1,55 × 1,14 (BLQ $1 \mathrm{n}^{\circ}$ 2173); F, m1 sen., 1,53 × 1,10 (BLQ $1 \mathrm{n}^{\circ}$ 2202); G, m2 sen., 1,49 × 1,27 (BLQ $\left.1 \mathrm{n}^{\circ} 2216\right) ; \mathbf{H}, \mathrm{m} 2$ sen., 1,41 × 1,13 (BLQ $1 \mathrm{n}^{\circ} 2221$ ); I, m2 sen., 1,43 × 1,21 (BLQ $1 \mathrm{n}^{\circ}$ 2222); J, M1 dex., 2,06 × 1,32 (BLQ $1 \mathrm{n}^{\circ} 2328$ ); K, M1 dex., 1,83 × 1,27 (BLQ $1 n^{\circ}$ 2335); L, M2 sen., 1,44 × 1,31 (BLQ $1 n^{\circ}$ 2383); M, M2 sen., 1,38 × 1,23 (BLQ 1 n $^{\circ} 2385$ ); N, M2 dex., 1,28 $\times 1,17$ (BLQ 1 $\mathrm{n}^{\circ}$ 2402); O, M2 sen., 1,48 × 1,30 (BLQ $\left.1 \mathrm{n}^{\circ} 2418\right) ; \mathbf{P}, \mathrm{m} 1$ dex., 1,74 × 1,32 (BLQ $\left.1 \mathrm{n}^{\circ} 2144\right) ; \mathbf{Q}, \mathrm{m} 2$ dex., 1,57 × 1,33 (BLQ $\left.1 \mathrm{n}^{\circ} 2152\right)$; R, M1 sen., 1,94 × 1,33 (BLQ $\left.1 \mathrm{n}^{\circ} 2154\right)$. Échelle: $1 \mathrm{~mm}$. 
TABleAU 4. - Mensurations (en $\mathrm{mm}$ ) des molaires de Democricetodon aff. gaillardi (Schaub, 1925) du gisement de Blanquatère 1 (Pyrénées-Orientales, France).

\begin{tabular}{|c|c|c|c|c|c|c|c|}
\hline & \multirow[b]{2}{*}{$\mathbf{N}$} & \multicolumn{3}{|c|}{ Longueur } & \multicolumn{3}{|c|}{ Largeur } \\
\hline & & $\min$ & moy & $\max$ & $\min$ & moy & $\max$ \\
\hline $\mathrm{m} 1$ & 4 & 1,61 & 1,68 & 1,74 & 1,19 & 1,24 & 1,32 \\
\hline $\mathrm{m} 2$ & 7 & 1,48 & 1,54 & 1,60 & 1,19 & 1,28 & 1,34 \\
\hline M1 & 6 & 1,74 & 1,89 & 1,98 & 1,23 & 1,31 & 1,39 \\
\hline M2 & 1 & & 1,53 & & & 1,35 & \\
\hline
\end{tabular}

m2. L'antérolophide labial est toujours bien développé alors que l'antérolophide lingual est quelquefois présent mais extrêmement réduit. Le mésolophide présente les mêmes variations que celles observées sur les $\mathrm{m} 1$. Le sinuside, dirigé vers l'avant, est bordé par une crête cingulaire plus marquée que sur $\mathrm{m} 1$.

m3. Réduite dans sa partie postéro-linguale, la partie antérieure présente les caractères de la $\mathrm{m} 2$.

Les molaires inférieures possèdent 2 racines.

\section{Molaires supérieures}

M1. Le développement du mésolophe est variable, il est le plus souvent de longueur moyenne mais peut aussi être plus long, atteignant quelquefois le bord labial. Il est en général plus long que le mésolophide de la $\mathrm{m} 1$. Le protolophule est simple, en position rétroverse ou double en proportions sensiblement équivalentes. Le métalophule est généralement rétroverse, délimitant un postéro-sinus plus ou moins réduit. Quelques dents montrent un métacône isolé. Une crête cingulaire obture presque toujours le sinus.

M2. Le protolophule est généralement double, mais lorsqu'il est simple, il peut être relié au protocône par la branche antérieure ou postérieure. Le mésolophe peut être court, moyen à long, atteignant même le bord labial; il est rarement absent. Le métalophule est le plus souvent en position proverse, relié au bras antérieur de l'hypocône, mais il peut être tranverse, rétroverse ou double. Le sinus est généralement bordé par une crête cingulaire assez forte qui se développe à partir du flanc de l'hypocône.
M3. Très réduite dans sa partie postérieure elle se caractérise par un antérolophe lingual toujours présent, plus ou moins développé, ainsi que par une crête linguale issue de l'hypocône extrêmement réduit bordant le sinus.

Les molaires supérieures présentent 3 racines.

\section{DISCUSSION}

La variation des dimensions des premières molaires inférieures et supérieures est nettement plus large que dans la population type de D. mutilus: Langenmoosen (Fahlbusch 1964). Les dimensions moyennes sont inférieures à celles de $D$. mutilus et bien plus petites que celles de D. affinis (Schaub, 1925) de La Grive M (mesures in Maridet 2002). C'est une caractéristique qui apparaît dans les importantes populations du Miocène moyen recueillies en Languedoc Roussillon (Aguilar et al. 1999), et elle devrait justifier la description d'une nouvelle espèce.

Cette abondante population de $D$. aff. mutilus de Blanquatère 1 fait connaître une grande variabilité morphologique dans laquelle on peut retrouver tous les caractères retenus par Fahlbusch (1964) pour les espèces D. mutilus et $D$. gracilis. La différence de taille est très nette entre ces deux espèces dans les gisements étudiés par Fahlbusch (1964) et elle est également marquée pour quelques dents à Blanquatère 1. Toutefois, compte tenu des dimensions de la population de Vieux-Collonges attribuée à $D$. gracilis, il apparaît délicat de discriminer avec certitude certaines des molaires de petite taille que nous avons affecté à $D$. aff. mutilus et $D$. gracilis.

\section{Democricetodon aff. gaillardi (Schaub, 1925)}

(Fig. 4P-R; Tableau 4)

Matériel et Dimensions. - Dents isolées (BLQ 1 no 2143 à 2159). Dimensions: voir Tableau 4.

\section{DESCRIPTION}

Molaires inférieures

Elles sont caractérisées par un mésolophide très développé, atteignant le bord lingual et pouvant développer un petit mésostylide. Le sinuside est 


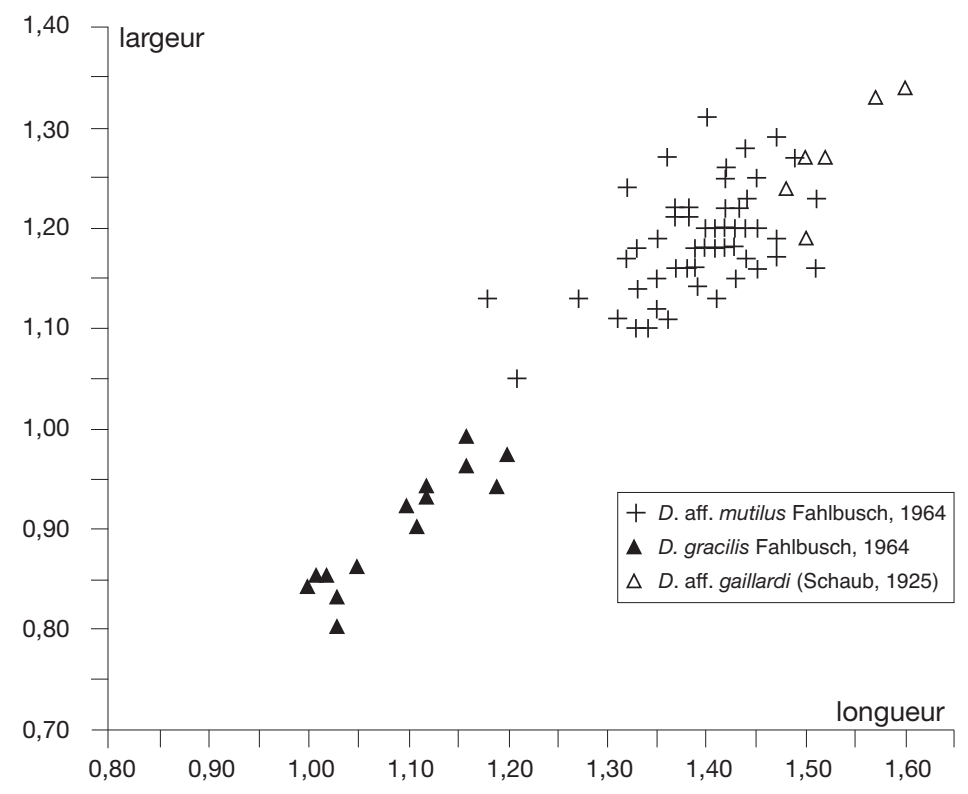

FIG. 5 - Diagramme bivarié de répartition longueur/largeur des mesures de la deuxième molaire inférieure (m2) des Democricetodon Fahlbusch, 1964 de Blanquatère 1 (Pyrénées-Orientales, France).

fermé par une crête labiale issue de la partie postéro-basale du protoconide tandis que le mésosinuside est obturé par une crête linguale issue de la partie postéro-basale du métaconide sur laquelle est éventuellement présent le mésostylide.

\section{Molaires supérieures}

Les M1 montrent un mésolophe bien développé qui n'atteint le bord lingual que sur deux dents. Il y a un double protolophule sur trois dents, le métacône est isolé sur une molaire, tandis que sur les autres le métalophule est rétroverse et délimite avec le postérolophe un petit postérosinus. Sur la M2 le mésolophe atteint le bord lingual, le protolophule est double et le métalophule rétroverse.

\section{DisCUSSION}

Ces molaires se distinguent des autres molaires de Democricetodon, présentes dans le gisement par un mésolophe(ide) plus développé en hauteur et longueur et des dimensions légèrement supérieures (Figs $3 ; 5$ ). En effet, chez $D$. aff. mutilus le mésolophide est rarement long et ne développe pas de mésostylide alors que ce caractère se retrouve chez $D$. gaillardi. En revanche, ces molaires se différencient de l'espèce $D$. gaillardi par une morphologie beaucoup plus simple: antérocone(ide) non dédoublé, mésostyle(ide) peu développé et absence d'ectomésolophe(ide), caractères généralement présents chez l'espèce de Sansan. Toutefois dans la population de D. gaillardi de Sansan, présente dans les collections de l'Université Montpellier 2, il existe quelques dents avec des morphotypes plus simples comparables à celles de Blanquatère 1. Comme les dimensions entrent dans le domaine de variation de celui des molaires de Sansan (Baudelot 1972; Maridet 2002) et que la morphologie simplifiée pourrait s'expliquer par l'âge plus ancien de Blanquatère 1 , nous attribuons ces quelques molaires à Democricetodon aff. gaillardi.

Famille EUCRICETODONTIDAE

Mein \& Freudenthal, 1971 
TABleau 5. - Mensurations (en $\mathrm{mm}$ ) des molaires de Eumyarion bifidus (Fahlbusch, 1964) du gisement de Blanquatère 1 (Pyrénées-Orientales, France).

\begin{tabular}{|c|c|c|c|c|c|c|c|}
\hline & \multirow[b]{2}{*}{$\mathbf{N}$} & \multicolumn{3}{|c|}{ Longueur } & \multicolumn{3}{|c|}{ Largeur } \\
\hline & & $\min$ & moy & $\max$ & $\min$ & moy & $\max$ \\
\hline $\mathrm{m} 1$ & 2 & 197 & & 2,08 & 1,14 & & 1,27 \\
\hline $\mathrm{m} 2$ & 5 & 1,42 & 1,51 & 1,64 & 1,12 & 1,20 & 1,26 \\
\hline m3 & 11 & 1,18 & 1,32 & 1,42 & 0,96 & 1,08 & 1,16 \\
\hline M1 & 2 & 1,92 & & 1,93 & 1,35 & & 1,38 \\
\hline M2 & 8 & 1,34 & 1,45 & 1,55 & 1,24 & 1,33 & 1,42 \\
\hline
\end{tabular}

Sous famille EUMYARIONINAE Unay, 1989 Genre Eumyarion Thaler, 1966

Eumyarion bifidus (Fahlbusch, 1964) (Fig. 6B-H; Tableau 5)

Matériel et Dimensions. - Dents isolées (BLQ 1 no 2 à 29). Dimensions: voir Tableau 5.

\section{DESCRIPTION}

\section{Molaires inférieures}

$\mathrm{m} 1$. L'antéroconide est relié à la première paire de tubercules par l'endolophide et l'antérolophulide; sur l'une des deux dents, l'antérolophulide rejoint l'endolophide, à la base de l'antéroconide, sur l'autre dent, ces deux crêtes sont bien distinctes. Sur une molaire, un éperon labial est présent dans le protosinuside alors que sur l'autre molaire, on observe une protubérance d'émail. Le bras postérieur du protoconide et le mésolophide sont bien développés et ils se rejoignent sur le bord lingual, délimitant un puiselet. Le bras postérieur de l'hypoconide est présent, mais sur une dent, compte tenu du degré d'usure, il tend à disparaître pour fusionner avec le postérolophide. L'ectomésolophide est large et bien développé, atteignant dans un cas le bord labial.

m2. L'antérolophide lingual est absent sur deux dents, plus ou moins développé sur les trois autres alors que l'antérolophide labial est toujours bien développé. Le bras postérieur du protoconide et le mésolophide présentent les mêmes caractéristiques que sur $\mathrm{m} 1$. L'ectomésolophide n'est visible que sur deux dents, mais il est nettement moins marqué que sur $\mathrm{m} 1$. Le bras postérieur de l'hypoconide est toujours présent.
Une molaire n'a pas de mésolophide et le bras postérieur de l'hypoconide est très réduit.

m3. Le bras postérieur du protoconide est toujours bien développé, atteignant le bord lingual alors que le mésolophide ne s'observe que sur deux dents et sous forme vestigiale. L'ectomésolophide n'est bien visible que sur 3 dents où il est moins développé que sur $\mathrm{m} 1$ et $\mathrm{m} 2$, alors que sur les autres molaires, il est absent ou à peine marqué. Le sinuside est transverse sur $\mathrm{m} 1$, transverse à rétroverse sur $\mathrm{m} 2$, un peu plus rétroverse sur $\mathrm{m} 3$.

\section{Molaires supérieures}

M1. L'antérocône est large avec deux facettes d'usure mais non divisé; l'antérolophule est double mais peu proéminent. Sur une dent, on observe un éperon labial très développé, atteignant le bord de la couronne. Le mésolophe est long, dans un cas, il atteint le bord labial, dans l'autre cas, il rejoint la partie labiale du métacone. Un entomésolophe peu développé n'est présent que sur une molaire.

M2. Le mésolophe est de longueur moyenne sur 2 dents; sur les autres, il est plus long, atteignant le bord labial; il peut s'unir à l'éperon postérieur du paracône (5 dents). Le protolophule est double sur 4 dents. Le métalophule est simple sauf sur une dent où il est double. Le sinus des M1-2 est nettement proverse.

\section{DISCUSSION}

Le genre Eumyarion est rarement représenté dans les gisements du sud de la France, 1 dent à Veyran (gisement palustre) (Thaler 1966; Aguilar 1980) et deux dents à Port-la-Nouvelle (gisement karstique) (Aguilar 1980) alors qu'il est bien représenté dans le gisement gersois de Sansan (Baudelot 1972) et dans de nombreux sites de Suisse (Engesser 1972; Bolliger 1992, 1994), d'Allemagne (Fahlbusch 1964; Wu 1982; Boon 1991) et de Tchécoslovaquie (Fejfar 1974).

Ces molaires de Blanquatère 1 different de celles de E. medius (Lartet, 1851) de Sansan par un aspect moins massif ainsi que par la présence du bras postérieur de l'hypoconide aux molaires inférieures. Elles sont en revanche comparables, aussi bien par les dimensions que par la morphologie, à celles de la population de E. bifidus de Puttenhausen décrite par Wu (1982). 

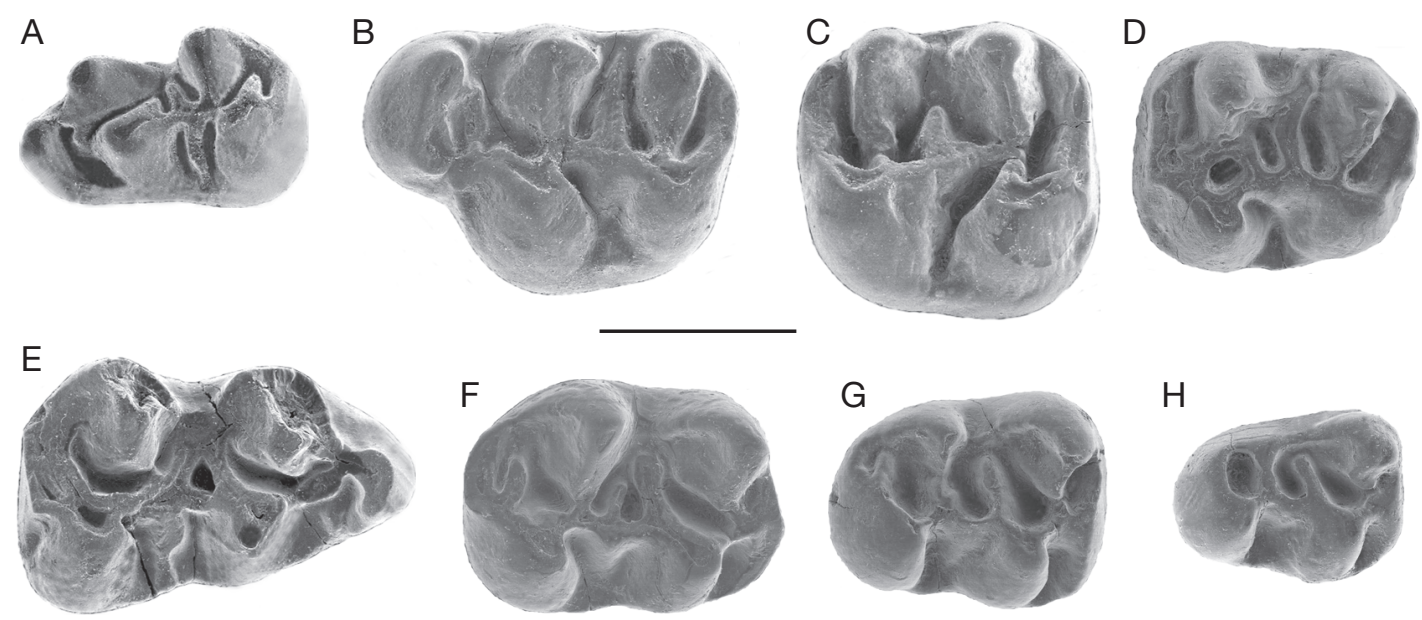

FIG. 6. - Molaires de Blanquatère 1 (mensurations en mm): A, Eumyarion sp., m1 sen., 1,47 × 0,86 (BLQ 1 n ${ }^{\circ}$ ); B-H, Eumyarion bifidus (Fahlbusch, 1964); B, M1 sen., 1,92 × 1,38 (BLQ $1 \mathrm{n}^{\circ}$ 20); C, M2 dex., 1,55 × 1,42 (BLQ $1 \mathrm{n}^{\circ} 22$ ); D, M2 sen., 1,46 × 1,30 (BLQ $1 \mathrm{n}^{\circ}$ 28); E, m1 dex., 2,08 × 1,27 (BLQ $1 \mathrm{n}^{\circ}$ 2); F, m2 dex., 1,64 × 1,21 (BLQ $1 \mathrm{n}^{\circ}$ 5); G, m3 dex., 1,42 × 1,16 (BLQ $\left.1 \mathrm{n}^{\circ} 15\right)$; H, m3 dex., 1,18 × 0,96 (BLQ $1 \mathrm{n}^{\circ}$ 14). Échelle: $1 \mathrm{~mm}$.

\section{Eumyarion sp.}

(Fig. 6A)

Matériel et dimensions. $-1 \mathrm{~m} 1(1,47 \times 0,86)$ (BLQ 1 $\left.\mathrm{n}^{\mathrm{o}} 1\right)$.

\section{DESCRIPTION}

Cette molaire est gracile avec des tubercules peu volumineux. L'antéroconide est petit, plus bas que les autres cuspides et bien distinct de la première paire de tubercule. Il est relié au métaconide par l'endolophide qui montre, près de la base du métaconide, un petit éperon dirigé vers l'avant. L'antérolophide labial est présent alors que l'antérolophide lingual est absent. Il n'y a pas de liaison entre le métaconide et le protoconide. Ce dernier développe un petit bras postérieur qui ne rejoint pas le mésolophide. Ce dernier est très développé mais n'atteint pas toutefois le bord lingual. L'ectomésolophide est fort et une crête cingulaire basse ferme le sinuside. L'hypoconide développe, dans le postérosinuside, une crête moyennement développée (bras postérieur) qui est issue du milieu de ce tubercule.

\section{DISCUSSION}

C'est la première fois qu'une espèce de petite taille $\mathrm{du}$ genre Eumyarion est signalée en Europe occidentale.
Les dimensions de cette molaire sont inférieures à celles des plus petites $\mathrm{m} 1$ connues à ce jour dans le gisement de Tägernaustrasse (Bolliger 1992) et dont la petite population est attribuée à l'espèce $E$. bifidus. Les seules espèces de petite taille attribuées au genre Eumyarion sont connues dans le Miocène inférieur de Turquie, dans les gisements de Harami 1, 3 et Keseköy (MN 2 ou 3), E. microps De Bruijn \& Sarac, 1991 et E. intercentralis De Bruijn \& Sarac, 1991, décrites par De Bruijn \& Sarac (1991). Par son antéroconide bien distinct de la première paire de tubercules, on ne peut rattacher cette molaire à E. intercentralis et l'absence de métalophulide I et II ne permet pas non plus de la rapprocher ni de E. intercentralis ni de E. microps.

Cette unique molaire et le peu de documentation sur les petites espèces du genre ne permettent pas de savoir si ce petit Eumyarion de Blanquatère 1 peut être le descendant de l'une des petites espèces du Miocène inférieur de Turquie. Il faut toutefois rappeler que le Megacricetodon tautavelensis Lazzari \& Aguilar, 2007 de Blanquatère 1 présente des ressemblances étroites avec la petite population turque de Cakaltepe attribuée par Sarica-Filoreau (2002) à $M$. aff. minor (Lartet, 1851) (Lazzari \& Aguilar 2007) ainsi qu'avec le Megacricetodon sp. du Miocène inférieur de Kesekoy (Wessels et al. 2001). 

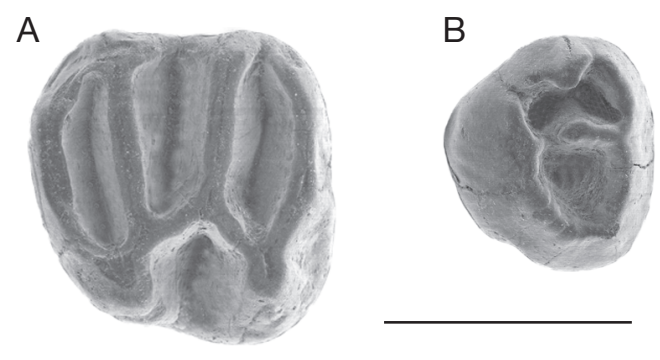

C
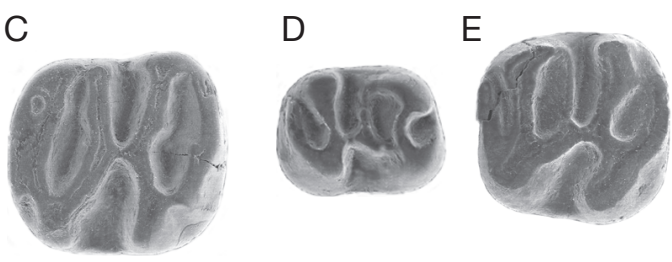

F

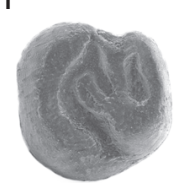

G
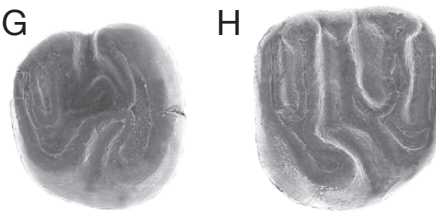

FIG. 7. - Molaires de Blanquatère 1 (Pyrénées-Orientales, France) (mensurations en $\mathrm{mm}$ ) : A, B, Ligerimys florancei Stehlin \& Schaub, 1951; A, M1 dex., 1,26 × 1,33 (BLQ $\left.1 \mathrm{n}^{\circ} 2504\right)$; B, M3 dex., $0,87 \times 1,01$ (BLQ $\left.1 n^{\circ} 2505\right) ; \mathbf{C}$, Ligerimys sp., m1 sen., 0,90 × 0,93 (BLQ $\left.1 n^{\circ} 2501\right)$; D-H, Keramidomys thaleri Hugueney \& Mein, 1968; D, p4 dex., 0,69 × 0,60 (BLQ $\left.1 \mathrm{n}^{\circ} 31\right)$; E, m1-2 sen., 0,80 × 0,81 (BLQ $\left.1 n^{\circ} 32\right)$; F, D4 dex., 0,64 × 0,71 (BLQ $1 n^{\circ} 34$ ); G, P4 sen., $0,73 \times 0,83\left(B L Q 1 n^{\circ} 35\right) ; \mathbf{H}, M 1-2$ sen., 0,78 $\times 0,94$ (BLQ 1 $\left.\mathrm{n}^{\circ} 38\right)$. Échelle: $1 \mathrm{~mm}$.

TABLEAU 6. - Mensurations (en mm) des molaires de Keramidomys thaleri Hugueney \& Mein, 1968 du gisement de Blanquatère 1 (Pyrénées-Orientales, France).

\begin{tabular}{lcccccccc}
\hline & & \multicolumn{3}{c}{ Longueur } & & \multicolumn{3}{c}{ Largeur } \\
\cline { 3 - 5 } \cline { 8 - 9 } & N & min & moy & $\max$ & & min & moy & $\max$ \\
\hline p4 & 2 & 0,69 & & 0,72 & & 0,60 & & 0,67 \\
m1-2 & 2 & 0,76 & & 0,80 & & 0,77 & & 0,81 \\
D4 & 1 & & 0,64 & & & 0,71 & \\
P4 & 2 & 0,71 & & 0,73 & & 0,82 & & 0,83 \\
M1-2 & 6 & 0,61 & 0,72 & 0,78 & & 0,78 & 0,86 & 0,94 \\
\hline
\end{tabular}

Famille EOMYiDae Depéret \& Douxami, 1902 Genre Ligerimys Stehlin \& Schaub, 1951
Ligerimys florancei Stehlin \& Schaub, 1951

(Fig. 7A, B)

Matériel et Dimensions. - 1 M1 $(1,26 \times 1,33) ; 1$ M3 $(0,87 \times 1,01)$ (BLQ 1 no $^{\circ} 2504$ et 2505$)$.

\section{DISCUSSION}

Ces deux molaires présentent une morphologie et des dimensions comparables à celles des populations décrites par Fahlbusch (1970) dans les gisements d'Allemagne, ainsi que celles des gisements du sud de la France (Escarguel \& Aguilar 1997).

\section{Ligerimys sp. (Fig. 7C)}

MatéRIel et dimensions. $-1 \mathrm{p} 4(0,77 \times 0,68) ; 1 \mathrm{~m} 1$ $(0,90 \times 0,93) ; 1 \mathrm{M} 1(0,80 \times 0,90) ; 1 \mathrm{M} 3(0,69 \times 0,85)$ (BLQ 1 no 2500 à 2503).

\section{DESCRIPTION}

La p4 montre un antérosinuside I moyennement développé mais peu profond. Le sinuside est en communication avec le mésosinuside. Sur m1, l'antérosinuside I est superficiel, dédoublé et disparait avec l'usure; la crête longitudinale est en position moyenne. La M2 ne possède pas de mésolophe et présente la particularité d'avoir un postérosinus divisé par une crête qui relie le postérolophe au métalophe. La M3 a une forme légèrement ovoide avec trois lophes.

\section{DisCUSSION}

Ces molaires montrent une morphologie que l'on retrouve chez L. antiquus Fahlbusch, 1970 des gisements du sud de la France (Escarguel \& Aguilar 1997), mais elles se distinguent des molaires de cette dernière espèce par des dimensions très petites. Ces dimensions sont inférieures aux plus petites espèces du genre connues à ce jour en Péninsule ibérique, L. ellipticus Daams, 1976 et L. palomae Alvarez Sierra, 1987 (Alvarez Sierra 1987). Les caractères morphologiques observés sur nos quatre dents ne sont pas caractéristiques de ceux décrits pour ces deux espèces ibériques, mais compte tenu de la variabilité morphologique observée et de notre matériel réduit, il n'est pas possible d'attribuer un nom spécifique à ce Ligerimys de Blanquatère 1 . 


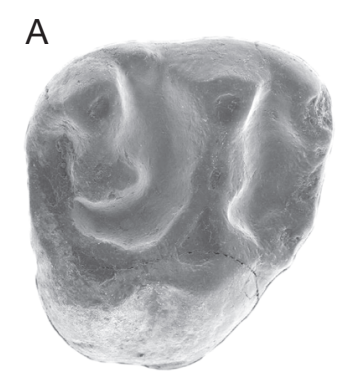

E

G

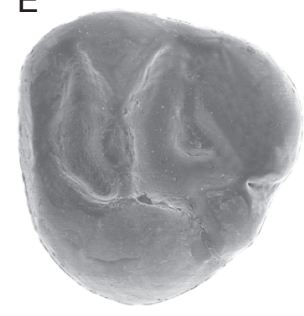

B

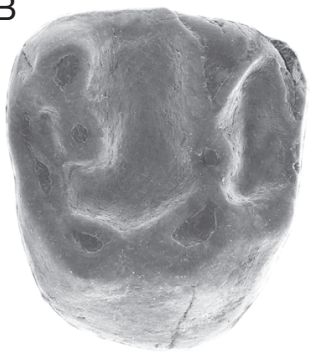

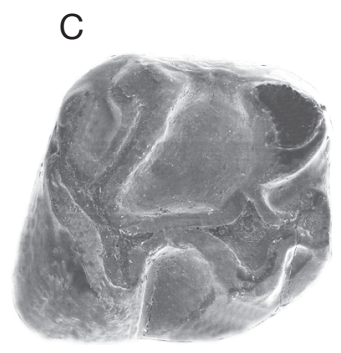
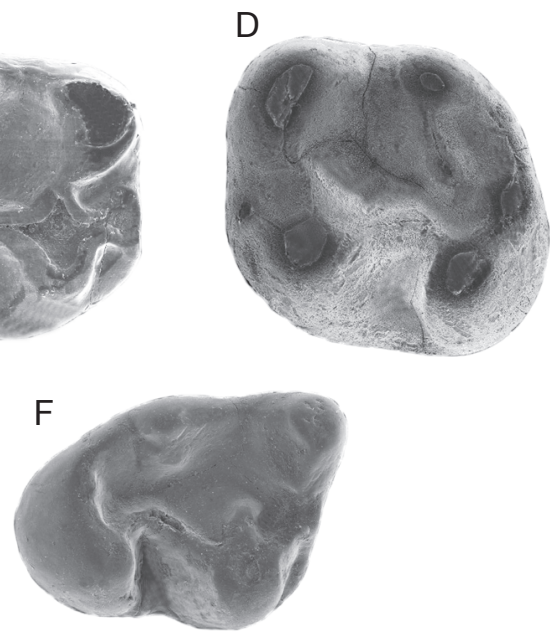

$\mathrm{H}$
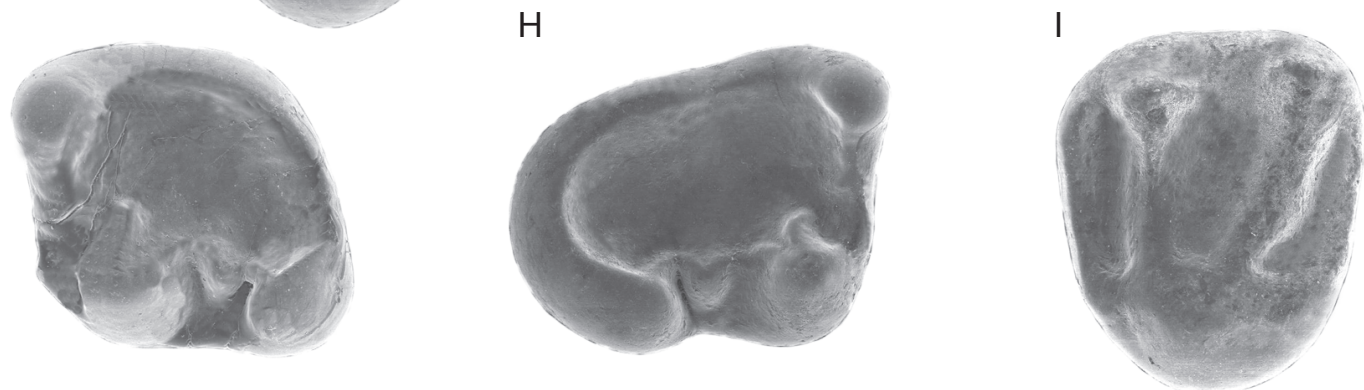

FIG. 8. - Molaires de Blanquatère 1 (Pyrénées-Orientales, France) (mensurations en mm): A-F, Heteroxerus rubricati Crusafont, de Villalta \& Truyols, 1955; A, M1 dex., 1,59 × 1,89 (BLQ $\left.1 \mathrm{n}^{\circ} 2108\right)$; B, M2 dex., 1,55 × 1,83 (BLQ $\left.1 \mathrm{n}^{\circ} 2111\right) ; \mathbf{C}, \mathrm{m} 1$ dex., 1,66 × 1,65 (BLQ $1 \mathrm{n}^{\circ}$ 2095); D, m2 sen., 1,66 × 1,40 (BLQ $\left.1 \mathrm{n}^{\circ} 2100\right)$; E, M3 sen., 1,46 × 1,59 (BLQ $1 \mathrm{n}^{\circ} 2114$ ); F, m3 dex., 1,71 × 1,68 (BLQ 1 $\mathrm{n}^{\circ}$ 2103); G-I, Spermophilinus bredai (von Meyer, 1848); G, m2 sen., 1,76 × 1,76 (BLQ $\left.1 \mathrm{n}^{\circ} 2073\right) ; \mathbf{H}, \mathrm{m}^{2}$ dex., 1,96 $\times 1,71$ (BLQ $1 \mathrm{n}^{\circ}$ 2077); I, M1-2 sen., 1,58 × 1,93 (BLQ $1 \mathrm{n}^{\circ}$ 2083). Échelle: $1 \mathrm{~mm}$.

Genre Keramidomys Hartenberger, 1967

Keramidomys thaleri Hugueney \& Mein, 1968 (Fig. 7D-H; Tableau 6)

Matériel et dimensions. - Dents isolées (BLQ 1 no 30 à 42). Dimensions: voir Tableau 6.

\section{DESCRIPTION}

\section{Denture inférieure}

p4. Les deux exemplaires montrent cinq lophides avec un synclinide I réduit. Le métalophide s'insère soit sur l'avant du protoconide, soit sur sa partie postérieure. Sur une dent, on note la présence de deux racines. m1-2. L'un des deux exemplaires montre quatre racines. Cinq lophides sont également présentes, l'antérolophide demeurant libre à son extrémité labiale. Le sinuside est rétroverse.

\section{Denture supérieure}

D4. Cette dent de petite dimensions présente une morphologie voisine de celle des P4 mais avec une partie postérieure très réduite et une absence de crête longitudinale.

P4. Ces deux dents présentent une morphologie différente, l'une montre une partie antérieure simplifiée comme on peut l'observer chez le spécimen type de l'espèce de Vieux-Collonges alors 
TABleau 7. - Mensurations (en mm) des molaires de Heteroxerus rubricati Crusafont, de Villalta \& Truyols, 1955 du gisement de Blanquatère 1 (Pyrénées-Orientales, France).

\begin{tabular}{|c|c|c|c|c|c|c|c|}
\hline & \multirow[b]{2}{*}{$\mathbf{N}$} & \multicolumn{3}{|c|}{ Longueur } & \multicolumn{3}{|c|}{ Largeur } \\
\hline & & $\min$ & moy & $\max$ & $\min$ & moy & $\max$ \\
\hline $\mathrm{d} 4$ & 2 & 1,17 & & 1,20 & 0,90 & & 0,93 \\
\hline p4 & 4 & 1,25 & 1,37 & 1,48 & 1,16 & 1,22 & 1,28 \\
\hline $\mathrm{m} 1$ & 4 & 1,59 & 1,63 & 1,66 & 1,56 & 1,61 & 1,65 \\
\hline $\mathrm{m} 2$ & 3 & 1,63 & 1,67 & 1,73 & 1,40 & 1,57 & 1,71 \\
\hline m3 & 3 & 1,71 & 1,74 & 1,76 & 1,61 & 1,68 & 1,74 \\
\hline D4 & 1 & & - & & & 1,29 & \\
\hline $\mathrm{P} 4$ & 1 & & 1,24 & & & 1,62 & \\
\hline M1 & 3 & 1,51 & 1,58 & 1,63 & 1,89 & 1,93 & 1,97 \\
\hline M2 & 5 & 1,55 & 1,58 & 1,60 & 1,83 & 1,91 & 2,04 \\
\hline M3 & 7 & 1,46 & 1,51 & 1,55 & 1,58 & 1,63 & 1,72 \\
\hline
\end{tabular}

TABleAU 8. - Mensurations (en $\mathrm{mm}$ ) des molaires de Spermophilinus bredai (von Meyer, 1848) du gisement de Blanquatère 1 (Pyrénées-Orientales, France). N, nombre de dents dont la longueur/largeur est mesurable.

\begin{tabular}{|c|c|c|c|c|c|c|c|}
\hline & \multirow[b]{2}{*}{$\mathbf{N}$} & \multicolumn{3}{|c|}{ Longueur } & \multicolumn{3}{|c|}{ Largeur } \\
\hline & & $\min$ & moy & $\max$ & $\min$ & moy & $\max$ \\
\hline $\mathrm{d} 4$ & $4 / 3$ & 1,25 & & 1,41 & 1,00 & & 1,18 \\
\hline $\mathrm{m} 2$ & 4 & 1,61 & & 1,76 & 1,71 & & 1,77 \\
\hline $\mathrm{m} 3$ & 2 & 1,96 & & 2,03 & 1,71 & & 1,72 \\
\hline D4 & $1 / 2$ & & 1,31 & & 1,25 & & 1,35 \\
\hline M1-2 & 5 & 1,57 & & 1,58 & 1,88 & & 1,93 \\
\hline M3 & 1 & & 1,78 & & & 1,70 & \\
\hline
\end{tabular}

que l'autre montre un schéma plus primitif. En effet, elle comporte un antérosinus très réduit, localisé sur le bord lingual et une ridule d'émail sur la face antérieure, au niveau du protocône. Sur une dent, le mésolophe est court, sur l'autre, il est plus développé mais n'atteint pas le bord lingual ni le paracône.

M1-2. Ces molaires possèdent trois racines et montrent un schéma occlusal avec cinq lophes bien développés. Le mésolophe est long, atteignant le bord lingual où il peut développer un mésostyle; il peut également rejoindre le paracône. Il n'y a pas de crête longitudinale. Le sinus dirigé vers l'avant est en communication avec le mésosinus.

\section{DISCUSSION}

Par leur morphologie et leurs dimensions, ces quelques dents sont comparables à celles de
K. thaleri, espèce connue à Vieux-Collonges (Hugueney \& Mein 1968) ainsi qu’à Vermes 1 (Engesser 1990).

Famille SCIURIDAE Gray, 1821

Sous-famille SCIURINAE Baird, 1857

Tribu XeRINI Osborn, 1910

Genre Heteroxerus Stehlin \& Schaub, 1951

\section{Heteroxerus rubricati}

Crusafont, de Villalta \& Truyols, 1955

(Fig. 8A-F; Tableau 7)

Matériel et Dimensions. - Dents isolées (BLQ 1 no 2088 à 2120). Dimensions: voir Tableau 7.

\section{DESCRIPTION}

\section{Molaires inférieures}

Le cingulum antérieur est plus ou moins développé. Les $\mathrm{m} 1$ montrent un fort antéroconulide. Le métalophide est complet sur deux $\mathrm{m} 1$ et deux $\mathrm{m} 2$.

\section{Molaires supérieures}

Les M1 et M2 montrent un protocône et un hypocône bien marqués, le protolophe est toujours relié au protocône et sur deux dents, il présente un petit protoconule. Le métalophe est plus ou moins plaqué contre le postérolophe et se termine par un fort métaconule.

\section{DisCUSSION}

Ces quelques molaires montrent des dimensions et une morphologie comparables à celles des populations des gisements ibériques, décrites par Cuenca Bescós (1988).

Tribu TAMIINI Black, 1963

Genre Spermophilinus De Bruijn \& Mein, 1968

Spermophilinus aff. bredai (von Meyer, 1848)

(Fig. 8G-I; Tableau 8)

MatéRiel et Dimensions. - Dents isolées (BLQ 1 $n^{\circ} 2069$ à 2086). Dimensions: voir Tableau 8. 


\section{DESCRIPTION}

Sur m2, l'antérolophide est libre sur une dent, alors que sur les autres il présente une fine liaison avec le protoconide; l'entoconide est réduit mais visible sur deux dents; le métalophide est résiduel sur une dent, complet sur les autres. Sur les M1-2, l'antérolophe est bas mais non isolé du protocône, le métaconule est inexistant.

\section{DisCUSSION}

Ces quelques molaires montrent des dimensions intermédiaires entre celles de $S$. besana Cuenca Bescós, 1988 représenté en Espagne et en France par quelques dents (Cuenca Bescós 1988; Aguilar 2002) et celles de S. bredai du gisement de Sansan (Baudelot 1972). Si l'on tient compte des caractères morphologiques énumérés par Cuenca Bescós (1988) qui permettent de différencier $S$. besana de $S$. bredai on s'aperçoit que c'est avec $S$. bredai que les dents de Blanquatère 1 présentent le plus d'affinité. Nous noterons que Cuenca Bescós (1988) signale pour $S$. besana un antérolophe isolé (sur la base de 3 dents) alors que la figure E, pl. 1 ne montre pas ce caractère.

Tribu Marmotini Pocok, 1923

Genre Palaeosciurus Pomel, 1853

?Palaeosciurus cf. fissurae Dehm, 1950

Matériel et dimensions. $-1 \mathrm{~m} 3(2,53 \times 2,34)$ (BLQ 1 no 2121).

\section{DISCUSSION}

Cette molaire est malheureusement très usée et son schéma dentaire n'est plus visible. Par ses grandes dimensions, elle peut être comparée aux $\mathrm{m} 3$ de $P$. fissurae du gisement de VieuxCollonges.

\section{Sciuridae indet.}

(Fig. 9)

Matériel ET Dimensions. $-1 \mathrm{~m} 2(1,40 \times 1,34)$; BLQ $1 \mathrm{n}^{\circ} 2087$.

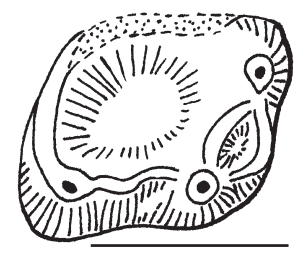

FIG. 9. - Molaire de Sciuridae indet. de Blanquatère 1 (PyrénéesOrientales, France). Échelle: $1 \mathrm{~mm}$.

\section{DESCRIPTION}

Cette molaire, de petite taille est très brachyodonte; elle montre une morphologie très simple, avec un protoconide et un métaconide reliés par l'antérolophide et le métalophide qui sont sensiblement à la même hauteur. Il n'y a pas d'antéroconulide. La partie postéro-linguale un peu abîmée ne permet pas d'affirmer l'absence d'entoconide. Il n'y a pas de mésoconide.

\section{Discussion}

La morphologie de cette molaire rappelle celle du genre Spermophilinus, mais en diffère par l'antérolophide et l'absence de mésoconide. Elle présente des dimensions inférieures à celles de $S$. besana, espèce la plus petite connue à ce jour (Cuenca Bescós 1988). Elle présente également une morphologie proche de celle du genre Freudenthalia Cuenca Bescós, 1988 dont deux espèces sont décrites dans des niveaux plus anciens: F. daamsi Cuenca Bescós, 1988 (Cuenca Bescós 1988) et F. cuencae Aguilar, 2002 (Aguilar 2002). Ces dernières ne sont représentées que par quelques spécimens et il est certain que nous ne connaissons pas parfaitement la variabilité morphologique. C'est avec $F$. cuencae que cette molaire présente le plus de ressemblance morphologique; elle en diffère toutefois par des dimensions inférieures.

\section{DISCUSSION}

Le prélèvement effectué à Blanquatère 1 correspond à un bloc de $60 \mathrm{~kg}$ environ d'argile indurée, extrait d'une fissure verticale de $1 \mathrm{~m}$ de large sur $3 \mathrm{~m}$ de hauteur. La faune recueillie est extrêmement riche en individus comme en témoignent plus de 6300 
TABleAu 9. - Liste faunique des rongeurs du gisement de Blanquatère 1 (Pyrénées-Orientales, France).

\section{Cricetidae Rochebrune, 1883}

Megacricetodon tautavelensis Lazzari \& Aguilar, 2007

Megacricetodon «collongensis-gersii » (Aguilar 1995)

Megacricetodon aunayi Lazzari \& Aguilar, 2007

Democricetodon aff. mutilus Fahlbusch, 1964

Democricetodon gracilis Fahlbusch, 1964

Democricetodon aff. gaillardi (Schaub, 1925)

Pseudofahlbuschia aff. jordensis

Freudenthal \& Daams, 1988

Eucricetodontidae Mein \& Freudenthal, 1971

Eumyarion bifidus (Fahlbusch, 1964)

Eumyarion sp.

Gerbillidae De Kay, 1842

Dakkamys sp.

Gliridae Thomas, 1897

Muscardinus sansaniensis (Lartet, 1851)

Glirudinus intermedius Aguilar \& Lazzari, 2006

Glirudinus magnus Aguilar \& Lazzari, 2006

Glirudinus cf. minutus Wu, 1993

Glirudinus cf. undosus Mayr, 1979

Microdyromys aff. koenigswaldi De Bruijn, 1966

Parglirulus werenfelsi Engesser, 1972

Miodyromys hamadryas (Forsyth Major, 1899)

Peridyromys murinus Pomel, 1853

Prodryomys remmerti Aguilar \& Lazzari, 2006

Prodryomys satus Mayr, 1979

Pseudodryomys ibericus De Bruijn, 1966

Pseudodryomys simplicidens De Bruijn, 1966

Vasseuromys aff. elegans Wu, 1993

Vasseuromys aff. priscus de Bonis, 1973

Eomyidae Depéret \& Douxami, 1902

Ligerimys florancei Stehlin \& Schaub, 1951

Ligerimys sp.

Keramidomys thaleri Hugueney \& Mein, 1968

Sciuridae Gray, 1821

Spermophilinus aff. bredai (von Meyer, 1848)

Heteroxerus rubricati Crusafont, de Villalta \& Truyols, 1955

?Palaeosciurus cf. fisurae Dehm, 1950

Sciuridae indet. (Freudenthalia?)

dents (premières et secondes molaires inférieures et supérieures) de rongeurs représentant 33 espèces (Tableau 9). Les représentants de la famille des Cricetidae sont de loin les plus abondants (5600 dents) (Fig. 10) et les représentants du genre Megacricetodon composent plus de la moitié de la faune comme cela est souvent le cas dans les gisements du Miocène moyen dans le sud de la France (Aguilar et al. 1999). Si le pourcentage des représentants de la famille des Gliridae est relativement faible par rapport à d'autres sites régionaux (Aguilar et al. 1999), il n'en demeure pas moins que cette famille est remarquable par sa richesse spécifique; en effet, 16 espèces y sont dénombrées (Aguilar \& Lazzari 2006) ce qui en fait la faune la plus diversifiée du sud de la France au cours du Miocène moyen. La présence de certaines de ces espèces témoigne de l'existence d'un couvert végétal non négligeable (Aguilar \& Lazzari 2006).

Cette richesse spécifique, souvent rencontrée dans des gisements karstiques, conduit les spécialistes à des points de vue contradictoires en rapport avec la biochronologie mammalienne, chaque fois que des associations d'espèces inconnues ou très rarement observées sont découvertes. En particulier, la reconnaissance d'associations fauniques non rencontrées dans des gisements d'origine lacustre ou palustre sensiblement contemporains a souvent jeté la suspicion quant à l'homogénéité de ces associations issues de remplissages, ainsi pour Castelnou 1b (Aguilar et al. 1991), Castelnou 6 (Aguilar et al. 1994), en Roussillon, ou VieuxCollonges (Bruijn et al. 1992) dans la Vallée du Rhône. Si le remplissage de certaines fissures karstiques est polyphasé dans quelques gisements du Languedoc-Roussillon, il a été montré que la procédure de sélection des blocs par analyse fine du sédiment permet de distinguer les phases du remplissage (Aguilar et al. 1996, 2007a, b ; Aguilar \& Michaux 1997 ; Sénégas et al. 2005).

Deux molaires uniques respectivement attribuées au Gerbillidae Dakkamys sp. (Lazzari et al. 2007) et à un Eumyarion de petite taille, forme mal connue en Europe occidentale, singularisent encore plus la faune de Blanquatère 1. Rappelons d'une part, que le Dakkamys sp. de Blanquatère 1 est le plus ancien représentant du genre, et qu'il présente de grandes ressemblances avec ceux que l'on retrouvera ensuite en Asie (Pakistan) (Lazzari et al. 2007) et d'autre part que les genres Megacricetodon et Eumyarion ne sont pas connus en Afrique. Il faut rappeler également que le Megacricetodon tautavelensis de ce même gisement, rappelle fortement les Megacricetodon de Turquie (Wessels 1996; Wessels et al. 2001 ; Sarica-Filoreau 2002). Tous ces éléments font que cette faune témoigne d'une migration, 
probablement consécutive à la fermeture orientale de la Téthys au cours du Langhien, phase migratoire dont la plus proche origine semble être la Turquie. La découverte récente parmi le matériel dentaire des molaires d'un insectivore inconnu en Europe (P. Mein comm. pers., étude en cours) pourrait appuyer cette hypothèse. L'association Ligerimys florancei-Keramidomys thaleri, trouvée pour la deuxième fois, après sa découverte à VieuxCollonges (Hugueney \& Mein 1968), gisement également d'origine karstique, singularise encore la faune de Blanquatère 1. La reconnaissance de cette association dans deux sites karstiques éloignés n'est pas sans conséquence sur la biochronologie mammalienne. D'une part, elle soutient la conclusion de Lazzari \& Aguilar (2007) sur l'homogénéité de la faune du gisement de Vieux-Collonges. D'autre part, elle ne permet plus de voir dans le genre Keramidomys Hartenberger, 1967, un marqueur de la biozone MN 5, et dans l'espèce Ligerimys florancei, une espèce caractéristique de la biozone MN 4. Ce dernier point souligne, s'il en était encore nécessaire, la difficulté de la définition des limites des zones MN.

\section{CONCLUSION}

Le gisement de Blanquatère 1 dont la position chronologique a été proposé à la limite MN 4/MN 5 avec un âge voisin de $16 \mathrm{Ma}$ (Lazzari \& Aguilar 2007) livre la plus riche faune de rongeurs du Miocène en Languedoc-Roussillon. Trente-trois espèces y sont dénombrées dont 16 pour la seule famille des Gliridae. La présence de taxons jusqu'alors inconnus en Europe occidentale (Dakkamys sp., Eumyarion sp. de petites dimensions) et d'espèces nouvelles, Megacricetodon tautavelensis, M. aunayi, Glirudinus intermedius Aguilar \& Lazzari, 2006, Prodryomys remmerti Aguilar \& Lazzari, 2006, indique une phase migratoire dont l'origine géographique la plus probable semble être le Proche-Orient, et la cause, la modification paléogéographique consécutive à la fermeture orientale de la Téthys au Langhien. Par sa richesse et ses particularités, le gisement de Blanquatère 1 représente une localité de référence pour le Miocène moyen en Europe sud-occidentale.

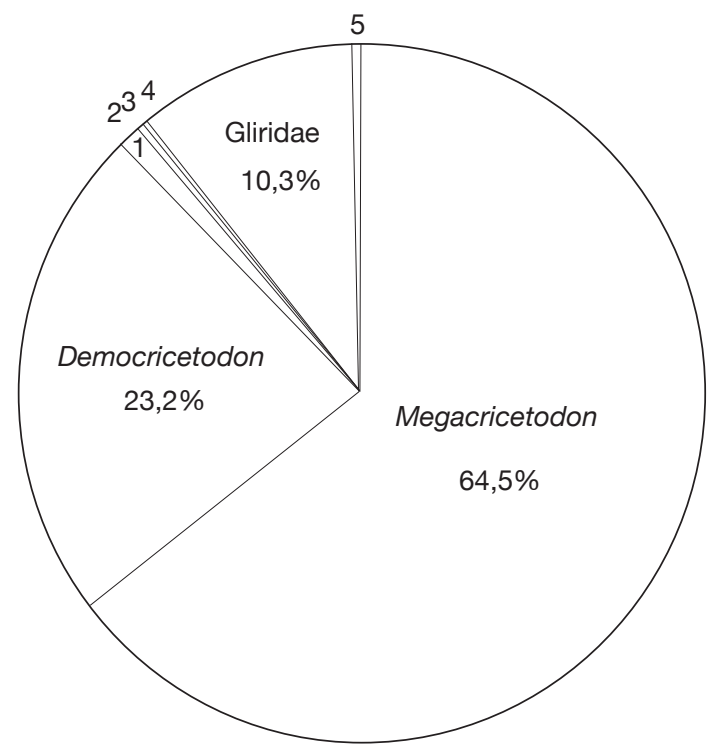

FIG. 10 - Représentation en pourcentage des familles et des genres de rongeurs du gisement de Blanquatère 1 (Pyrénées-Orientales, France). Le pourcentage est établi à partir du nombre total des deux premières molaires inférieures et supérieures: 1, Pseudofahlbuschia Freudenthal \& Daams, 1988, 1,1 \%; 2, Eumyarion Thaler, 1966, 0,3 \% ; 3, Gerbillidae De Kay, 1842, 0,02 \% ; 4, Eomyidae Depéret et Douxami, 1902, 0,2 \%; 5, Sciuridae Gray, 1821, 0,4 \%.

\section{Remerciements}

Nous remercions les rapporteurs P. Mein (Université Claude Bernard, Lyon 1) et S. Sen (Muséum national d'Histoire naturelle, Paris) pour leurs remarques constructives.

Publication ISE-M-2010 - 014 (UMR 5554, CNRS).

\section{RÉFÉRENCES}

Aguilar J.-P. 1980. — Rongeurs du Miocène inférieur et moyen en Languedoc. Leur apport pour les corrélations marin-continental et la stratigraphie. Palaeovertebrata 9 (6): 155-203.

AgUILAR J.-P. 1981. - Évolution des rongeurs miocènes et paléogéographie de la Méditerranée occidentale. Thèse Science, Université Montpellier II, France, 203 p.

Aguilar J.-P. 1995. - Évolution de la lignée Megacricetodon collongensis-Megacricetodon roussillonensis (Cricetidae, Rodentia, Mammalia) dans le Sud de la France. Palaeovertebrata 24 (1): 1-45.

Aguilar J.-P. 1999. — Les rongeurs de deux sites kars- 
tiques du Miocène moyen des Pyrénées-Orientales (Sud de la France) avec une nouvelle espèce de Pseudofalbbuschia (Rodentia, Mammalia). Eclogae Geologicae Helvetiae 92: 475-481.

Aguilar J.-P. 2002. - Les sciuridés des gisements karstiques du Miocène inférieur à moyen du sud de la France: nouvelles espèces, phylogénie, paléoenvironnement. Geobios 35: 375-394.

Aguilar J.-P. \& LAZZARI V. 2006. — Les gliridés du gisement karstique de Blanquatère 1 (Miocène moyen, Sud de la France) : espèces nouvelles. Geodiversitas 28 (2): 277-295.

Aguilar J.-P. \& Michaux J. 1997. — Les faunes karstiques néogènes du Sud de la France et la question de leur homogénéité chronologique, in Aguilar J.-P., Legendre S. \& Michaux J. (eds), Biochronologie mammalienne du Cénozoïque en Europe et domaines reliés. Actes du Congrès Biochrom'97. Mémoires et Travaux de l'École pratique des Hautes Etudes, Institut de Montpellier, Sciences de la Vie et de la Terre 21: 33-38.

Aguilar J.-P., Calvet M. \& Michaux J. 1991. — Présence de Progonomys (Muridae, Rodentia, Mammalia) dans une association de rongeurs de la fin du Miocène moyen (Castelnou 1B, Pyrénées-Orientales, France). Geobios 24 (4): 503-508.

Aguilar J.-P., Calvet M. \& Michaux J. 1994. — Les rongeurs de Castelnou 6 (Pyrénées-Orientales, France) et les corrélations entre faunes ibériques et françaises au Miocène moyen. Neues Jahrbuch für Geologie und Paleontologie, Monatshefte 192 (1): 109-131.

Aguilar J.-P., Escarguel G., Crochet J.-Y., Sigé B. \& SUdRE J. 1996. — Nouveaux sites d'âges variés dans les remplissages karstiques du Miocène inférieur de Bouzigues (Hérault, Sud de la France). Part. II : faunes 2 (Rongeurs), biochronologie et corrélations. Geobios M.S. 20: 485-491.

Aguilar J.-P., Escarguel G. \& Michaux J. 1999. A succession of Miocene rodent assemblages from fissure fillings in southern France: plaeoenvironmental interpretation and comparison with Spain. Palaeogeography, Paleaoclimatology, Paleaoecology 145: 215-230.

Aguilar J.-P., Lazzari V., Michaux J., Sabatier M. \& Calvet M. 2007a. - Lo Fournas 16-M (Miocène supérieur) et Lo Fournas 16-P (Pliocène moyen), deux nouvelles localités karstiques à Baixas, Sud de la France. Part. I: description et implications géodynamiques. Géologie de la France 1: 55-62.

AgUilar J.-P., Michaux J. \& LAZZARI V. 2007b. — Lo Fournas 16-M (Miocène supérieur) et Lo Fournas 16-P (Pliocène moyen), deux nouvelles localités karstiques à Baixas, Sud de la France. Part II : les faunes de rongeurs et nouvelles espèces. Géologie de la France 1: 63-81.

Alvarez Sierra M. A. 1987. - Estudio sistemático y bioestratigráfico de los Eomyidae (Rodentia) del Oligoceno superior y Mioceno inferior español. Scripta Geologica 86: 1-207.

BAUdELOT S. 1972. - Étude des chiroptères, insectivores et rongeurs du Miocène de Sansan (Gers). Toulouse, Thèse, Université P. Sabatier, France, 364 p.

Bolliger T. 1992. - Kleinsäuger aus der Miozänmolasse der Ostschweiz. Documenta naturae München 75: 1-296.

Bolliger T. 1994. - Die Obere Süsswassermolasse in Bayern und der Ostschweiz: bio- und lithostratigraphische Korrelationen. Mitteilungen der Bayerischen Staatssamlung für Paläontologie und Historische Geologie, München 34: 109-144.

Boon E. 1991. - Die Cricetiden und Sciuriden der Oberen Süsswasser-molasse von Bayerisch-Schwaben und ihre stratigraphische Bedeutung. Inaugural Dissertation, München, 143 p.

Bruijn H. DE \& SARAC G. 1991. - Early Miocene rodent faunas from the eastern Mediterranean area. Part I. The genus Eumyarion. Proceedings of the Koninklijke Nederlandse Akademie Van Wetenschappen B, 94 (1): 1-36.

Bruijn H. De, DaAms R., DaXner-Höck G., Fahlbusch V., Ginsburg L., Mein P. \& Morales J. 1992. - Report of the RCMNS working group on fossil mammals, Reisensburg 1990. Newsletters on Stratigraphy 26: 65-118.

Cuenca BesCós G. 1988. — Revision de los Sciuridae del Aragoniense y del Rambliense en la fosa de Calatayud-Montalban. Scripta Geologica 87: 1-116.

DAAMS R. 1999. - Family Gliridae, in RÖSSNER G. \& HeIssig K. (eds), The Miocene Land Mammals of Europe. F. Pfeil, Munich: 301-318.

ENGESSER B. 1972. —Die obermiozäne Sängetierfauna von Anwil (Baselland). Tätigkeitsbericht der Naturforschenden Gesellschaft, Baselland 28: 37-363.

ENGESSER B. 1990. - Die Eomyidae (Rodentia Mammalia) der Molasse der Schweiz und Savoyens. Systematik und Biostratigraphie. Mémoires suisses de Paléontologie 112: 1-144.

EsCARGUel G. \& Aguilar J.-P. 1997. — Les éomyidés Pseudotheridomys et Ligerimys (Rodentia, Mammalia) du Miocène inférieur du Sud de la France. Évolution et biostratigraphie. Palaeontographica $247(1-4): 25-58$

Fahlbusch V. 1964 - Die Cricetiden der oberen Süsswassermolasse Bayerns. Bayerische Akademie der Wissenschaften, Mathematisch-naturwissenschaftliche Klasse, München, Abhandlungen N.F. 118, 136 p.

FAHLBUSCH V. 1970. — Populationsverschiebungen bei tertiären Nagetieren, eine Studie an oligozänen und miozänen Eomyidae Europas. Abhandlungen der Bayerischen Akademie der Wissenschaften, Mathematisch-naturwissenschaftliche Klasse, N.F., München 145: 1-136. 
FEJFAR O. 1974. — Die Eomyiden und Cricetiden (Rodentia Mammalia) des Miozäns der Tschechoslowakei. Paleontographica 146 (4-6): 100-180.

Freudenthal M. 2006. - The status of Democricetodon, Fahlbuschia, Pseudofahlbuschia and Renzimys (Cricetidae, Mammalia). A reply to Van der Meulen et al. (2004). Geobios 39: 43-55.

FreUdenthal M. \& DAAMS R. 1988. - Cricetidae (Rodentia) from the type-Aragonian; the genera Democricetodon, Fablbuschia, Pseudofahlbuschia nov. gen., and Renzimys, in Freudenthal M. (ed.), Biostratigraphy and paleoecology of the Neogene micromammalian faunas from the CalatayudTeruel Basin (Spain). Scripta Geologica Special Issue 1: 133-252.

Hugueney M. \& Mein P. 1968. — Les Eomyidés (Mamalia, Rodentia) néogènes de la région lyonnaise. Geobios 1: 187-204.

LazZari V. \& Aguilar J.-P. 2007. - Les Megacricetodon du gisement karstique Miocène moyen de Blanquatère 1 (Pyrénées-Orientales, Sud de la France) : nouvelles espèces, implications biochronologique et phylogénique. Geobios 40: 91-111.

LAZZARI V., MichauX J. \& Aguilar J.-P. 2007. First occurence in Europe of Myocricetodontinae (Rodentia, F) during the Lower Middle Miocene in the karstic locality of Blanquatère 1 (Southern France): implications. Journal of Vertebrate Paleontology 27 (4): 1062-1065.

MARIDET O. 2002. - Révision du genre Democricetodon (Mammalia, Rodentia, Cricetinae) et dynamique des faunes de rongeurs du Néogène d'Europe occidentale : évolution, paléobiodiversité et paléobiogéographie. Thèse, Université Claude Bernard, Lyon I, France, 252 p.

Mein P. \& Freudenthal M. 1971. - Les Cricetidae (Mammalia, Rodentia) du Neogène moyen du Vieux-Collonges. Partie $1:$ le genre Cricetodon Lartet, 1851. Scripta Geologica 5: 1-51.

Moser M., RÖssner G.E., GÖHLICH U. B., BÖHME M. \& FahlBUSCH V. 2009. — The fossil lagerstätte
Sandelzhausen (Miocene; southern Germany): history of investigation, geology, fauna, and age. Paläontologische Zeitschrift 83: 7-23.

SARICA-FILOREAU N. 2002. - Faunes de rongeurs néogènes et quaternaires des grabens d'Anatolie occidentale. Systématique, biochronologie et implications tectoniques. Thèse, Muséum national d'Histoire naturelle, Paris, France, 345 p.

Sénégas F., Paradis E. \& Michaux J. 2005. - Homogeneity of fossil assemblages extracted from mine dumps: an analysis of Plio-Pleistocene fauna from South African caves. Lethaia 38 (4): 315-322.

THALER L. 1966. - Les rongeurs fossiles du BasLanguedoc dans leurs rapports avec l'histoire des faunes et la stratigraphie du Tertiaire d'Europe. Mémoires du Muséum national d'Histoire naturelle, Paris, sér. C (17): 1-295.

Van der Meulen A. J. \& DaAms R. 1992. - Evolution of Early-Middle Miocene rodent faunas in relation to long-term paleoenvironmental changes. Palaeogeography, Palaeoclimatology, Palaeoecology 93: 227-253.

Van der Meulen A., Pelaez-Campomanes P. \& DAAMS R. 2004. - Revision of medium-sized Cricetidae from the Miocene of the Daroca-Villafeliche area in the Calatayud-Teruel basin (Zaragoza, Spain). Coloquios de Paleontologia Vol. Extra 1: 385-441.

WESSELS W. 1996. - Myocricetodontinae from the Miocene of Pakistan. Proceedings of the Koninklijke Nederlandse Akademie Van Wetenschappen B 99 (3-4): 253-312.

Wessels W., Theocharopoulos H., De Bruijn H. \& ÜNAY E. 2001. - Myocricetodontinae and Megecricetodontini (Rodentia) from the Lower Miocene of NW Anatolia. Lynx 32: 371-388.

WU W. 1982. - Die Cricetiden (Mammalia, Rodentia) aus der Oberen Süsswasser- Molasse von Puttenhausen (Niederbayern). Mitteilungen der Bayerischen Staatssamlung für Paläontologie und Historische Geologie, München 30: 65-105. 